Cerebrovasc Dis 2009;27:309-310

DOI: $10.1159 / 000201559$

\section{Spontaneous Cervical Artery Dissection in Adult Williams Syndrome}

\author{
Peter Vanacker ${ }^{\mathrm{a}}$, Vincent Thijs ${ }^{\mathrm{a}, \mathrm{b}}$ \\ ${ }^{a}$ Department of Clinical and Experimental Neurology, \\ University Hospitals, 'besalius Research Center, VIB, Leuven, \\ Belgium
}

Williams syndrome (WS), a rare neurodevelopmental disorder, is caused by microdeletion at chromosomal subunit 7q11.23, encompassing elastin (ELN) in $90 \%$ of the cases [1]. WS is characterized by cardiovascular and endocrine abnormalities, elfinlike facial appearance and mental retardation. Many of the cardiovascular features in WS are the result of ELN haploinsufficiency. Developmental changes in the vessel wall in WS are associated with altered vessel compliance and increased mean arterial pressure [2]. Although stroke is rare in WS, vascular disease, especially supravalvular aortic stenosis and pulmonary artery stenosis, is a classical complication (>70\%). Many of the cardiovascular abnormalities found in WS are established risk factors for stroke, including arterial hypertension and cardiac disease [3]. Progressive intracranial stenosis leading to moyamoya phenomena has also been reported in a few cases [3]. Given its major role in the structure of the vessel wall, ELN is also a target of investigation for the identification of the etiology of spontaneous cervical artery dissection (CAD) [4]. ELN mutations have, however, not been found in patients with CAD [5].

A 51-year-old woman, with typical morphological characteristics and FISH confirmed deletion of 7q11.23, was admitted for acute left hemiplegia. Genetic analysis was performed in 1997. Physical examination revealed complete left hemiplegia, visual and tactile neglect and homonymous hemianopsia. There was no history of trauma or risk factors for stroke, except for well-controlled hypertension.

Diffusion-weighted MRI showed a recent infarction in the middle cerebral artery region (fig. 1A). On carotid Doppler ultrasonography and CT angiography, a flame-shaped occlusion $1 \mathrm{~cm}$ after the origin of the right internal carotid artery (ICA) was found (fig. 1B). The left ICA showed a sudden caliber reduction at the cervical level for more than a length of $1 \mathrm{~cm}$ (fig. 1D). Two months later, a control CT angiography revealed recanalization of the right ICA (fig. 1B) and formation of pseudoaneurysm at the left ICA (fig. 1E). Further cardiovascular workup revealed a moderate aortic cusp insufficiency.

To our knowledge, this is the first report of spontaneous CAD in a patient with WS. So far the literature only describes stenoses of medium- and large-sized arteries as the origin of stroke in WS [3]. But, based on this case report, we conclude that stroke in WS may be related to a CAD. We assume that the dissection is caused by an ELN arteriopathy leading to hypertension, increased arterial stiffness and vessel wall stress. It is known that ELN haploinsufficiency leads to an in increased proliferation of vascular smooth muscle cells, thicker intima-media thickness and arterial vasculopathy with luminal narrowing [7]. Electron microscopy showed major elastic fiber abnormalities with renal artery stenosis in WS, and pathologic observations showed thick irregular elastic fibers [8]. In mouse models of WS with ELN deficiency, obstructive arterial disease is accompanied by a compensatory increase in the number of rings of elastic lamellae and by intimal smooth muscle proliferation and reorganization [9]. In addition, previous studies demonstrated mild elastic fiber abnormalities in a third of the spontaneous CADs. Using electron microscopic investigation of skin biopsies, previous studies also revealed the presence of abnormalities in the connective tissue morphology in $50-60 \%$ of these patients [6]. Nevertheless, new studies are needed to discuss the role of WS in the group of genetic connective tissue diseases which predisposes to CAD.

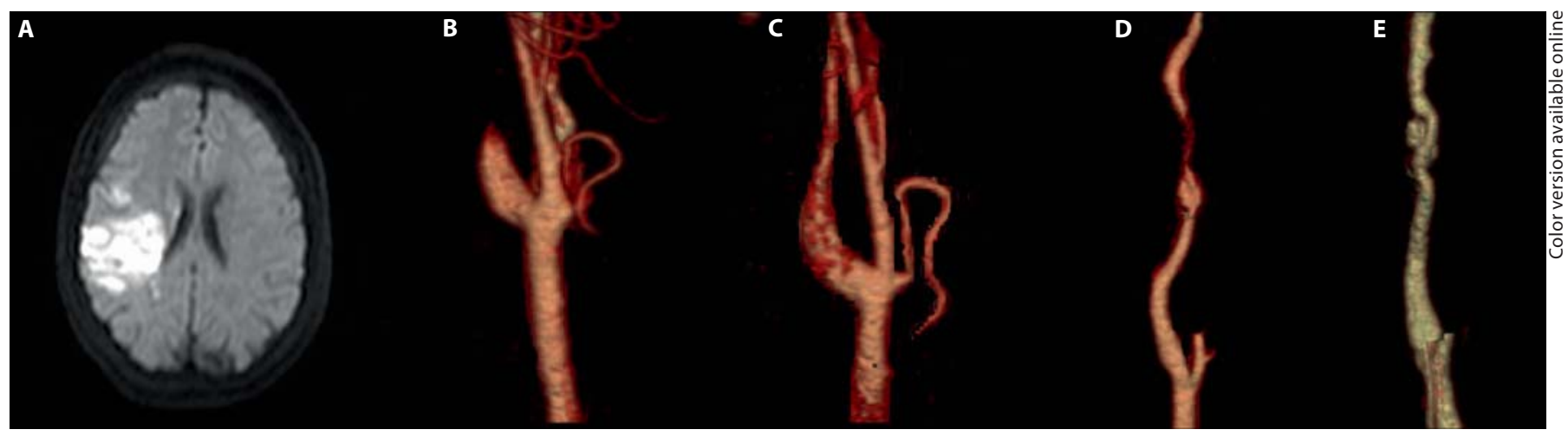

Fig. 1. Cerebral imaging of a spontaneous cervical artery dissection in a patient with Williams syndrome. A Diffusion-weighted MRI showing a recent middle cerebral artery infarct. B-E CT angiography visualized post-ostial dissection of the right internal carotid artery (ICA, B) with recanalization 2 months later (C) and a caliber reduction at the cervical level of left ICA (D), later on taking shape of a pseudoaneurysm (E). 


\section{References}

$\checkmark 1$ Pober BR, Morris CA: Diagnosis and management of medical problems in adults with Williams-Beuren syndrome. Am J Med Genet C Semin Med Genet 2007;145C:280-290.

-2 Faury G, Pezet M, Knutsen RH, Boyle WA, Heximer SP, McLean SE Minkes RK, Blumer KJ, Kovacs A, Kelly DP, Li DY, Starcher B, Mecham RP: Developmental adaptation of the mouse cardiovascular system to elastin haploinsufficiency. J Clin Invest 2003;112:1419-1428.

3 Wollack JB, Kaifer M, LaMonte MP, Rothman M: Stroke in Williams syndrome. Stroke 1996;27:143-146.

$\checkmark 4$ Rubinstein SM, Peerdeman SM, Van Tulder MW, Riphagen I, Haldeman S: A systematic review of the risk factors for cervical artery dissection. Stroke 2005;36:1575-1580.

5 Grond-Ginsbach C, Thomas-Feles C, Werner I, Weber R, Wigger F, Hausser I, Brandt T: Mutations in the tropoelastin gene (ELN) were not found in patients with spontaneous cervical artery dissection. Stroke 2000;31:1935-1938.
-6 Pober BR, Johnson M, Urban Z: Mechanisms and treatment of cardiovascular disease in Williams-Beuren syndrome. J Clin Invest 2008;118: 1606-1615

7 Sadler LS, Gingell R, Martin DJ: Carotid ultrasound examination in Williams syndrome. J Pediatr 1998;132:354-356.

8 Lacolley P, Boutouyrie P, Glukhova M, Daniel Lamaziere JM, Plouin PF, Bruneval P, Vuong P, Corvol P, Laurent S: Disruption of the elastin gene in adult Williams syndrome is accompanied by a paradoxical reduction in arterial stiffness. Clin Sci (Lond) 2002;103:21-28.

9 D'Armiento J: Decreased elastin in vessel walls puts the pressure on. J Clin Invest 2003;112:1308-1310.

Peter Vanacker, MD

Department of Clinical and Experimental Neurology

University Hospitals, BE-3000 Leuven (Belgium)

Tel. +32 1634 4280, Fax +32 16344285

E-Mail peter.vanacker@uzleuven.be 